Published in final edited form as:

Trends Endocrinol Metab. 2018 May ; 29(5): 349-359. doi:10.1016/j.tem.2018.03.002.

\title{
What can 'brown-ing' do for you?
}

\author{
Amar M. Singh and Stephen Dalton* \\ Center for Molecular Medicine, Department of Biochemistry and Molecular Biology, University of \\ Georgia, 325 Riverbend Road, Athens, GA 30602
}

\begin{abstract}
Human stem cell-based models of thermogenic adipocytes provide an opportunity for the establishment of new therapeutics, modeling disease mechanisms and understanding development. Pluripotent stem cells, adipose-derived stem cells/pre-adipocytes and programming/reprogramming-based approaches have been used to develop cell-based platforms for drug screening and transplantable therapeutics in the metabolic disease arena. Here, we will provide a detailed overview of these approaches, the latest advances in this field and the opportunities and shortcomings they present. Moreover, we will comment on how stem-cell based platforms can be best utilized in the future for the treatment and understanding of metabolic diseases, including type 2 diabetes and associated medical issues such as obesity.
\end{abstract}

\section{Keywords}

beige fat; brown fat; pluripotent stem cells; mesenchymal stem cells; adipose-derived stem cells; metabolic disease

\section{Cellular function of brown and beige fat}

Classical brown fat exhibits distinct characteristics that distinguish it from the more abundant depots of white fat, such as the expression of uncoupling protein-1 (UCP1; Box 1) [1]. Early studies indicate that the prevalence of brown fat in humans declines during ageing and is unlikely to be important later in life [2]. More recent studies, however, show that adult humans have functional brown fat depots that can be thermogenically-activated following cold exposure [3]. Using positron emission tomography and X-ray computed tomography (PET/CT) scans, fat depots capable of cold-stimulated glucose uptake have been identified in humans. Upon histological analyses of these fat depots, $\mathrm{UCP}^{+}$adipocytes were identified, confirming the persistence of brown adipose tissue in human adults [4-7]. Importantly, a clear inverse correlation exists between body mass index and the amount of brown adipose tissue (BAT). This implicates brown fat as being an important tissue that may prevent the development of obesity and obesity-related metabolic diseases such as diabetes.

*Correspondence: sdalton@uga.edu (S. Dalton).

Publisher's Disclaimer: This is a PDF file of an unedited manuscript that has been accepted for publication. As a service to our customers we are providing this early version of the manuscript. The manuscript will undergo copyediting, typesetting, and review of the resulting proof before it is published in its final citable form. Please note that during the production process errors may be discovered which could affect the content, and all legal disclaimers that apply to the journal pertain. 
Recently, thermogenic adipocytes have been identified in white adipose tissue (WAT). These cells are generally referred to as 'beige' or 'brite' (for brown-in-white) adipose cells, from herein referred to as beige cells (Box 1) [8-10]. Like brown fat, beige fat cells are also defined by the expression and functional activity of UCP1. Furthermore, the transcriptional networks that regulate brown and beige adipocytes appear to be similar with C/EBP $\beta$, PGC1a and EBF2 playing important roles in their specification and/or maintenance of identity. For further reading on the transcriptional networks that control brown fat and beige fat, several other reviews have been recently published [11, 12]. Importantly, beige and brown adipocytes have the potential to modulate disease progression by serving as a "metabolic sink" (Box 2). Here, thermogenic adipocytes could serve to modulate circulating metabolite levels that impact systemic metabolism that impacts diabetes and obesity. Therapeutically useful thermogenic adipocytes activity could potentially be generated by the differentiation/activation of endogenous adipose-derived progenitor cells or, through the transplantation of exogenous cells derived from different potential sources (see Figure).

Another important feature of thermogenic adipocytes is their ability to function as a secretory organ [13]. Numerous reports indicate that BAT performs an endocrine function that impacts other tissues including WAT, liver, pancreas and the immune system. Several physiologically-relevant cytokines are secreted by BAT ('batokines'), including VEGF and potentially FGF21 [3]. FGF21 is expressed at high levels in BAT from mice and humans and has been linked to correspondingly elevated levels of FGF21 in the circulation. This link however, is made more complicated by other studies indicating that FGF21 is also secreted by the liver [14]. FGF21 functions by regulating glucose and lipid metabolism in the liver which serves to stimulate activity of the sympathetic nervous system and by promoting the browning of WAT. BAT also secretes exosome and non-exosome vesicles that can potentially regulate systemic metabolism $[15,16]$. For example, Kahn and colleagues identified miRNAs in BAT-derived exosomes that regulate circulating FGF21 and hepatic FGF21 production, subsequently resulting in increased glucose tolerance [16]. The identification and function of 'batokines' in the modulation of metabolic homeostasis further supports the concept that activation of thermogenic adipocytes could serve as a therapeutic for treatment of metabolic diseases. In summary, thermogenic adipocytes could have therapeutic potential following transplantation into patients with metabolic diseases or, in drug screening approaches to identify compounds that modulate thermogenic activity in the body. In the following sections, we will describe the cell sources and culture systems for derivation of thermogenic adipocytes and potential opportunities for their therapeutic use.

\section{Human thermogenic adipocyte cell culture models}

Several cell culture platforms can be potentially utilized for modeling human thermogenic adipocytes (Table 1). The first option utilizes the directed-differentiation of human pluripotent stem cells (hPSCs) or adult stem cells (hASCs), where specific chemicals and growth factors promote their differentiation into a thermogenic state that reproduces the embryonic development of brown adipocytes. A second approach involves the conversion of cells in the white adipocyte lineage, including MSCs and adipose derived stem cells, to a thermogenic identity using small molecules and/or growth factors. Finally, ectopic overexpression of transcription factors involved in development along the thermogenic cell 
program could be potentially used to drive differentiation or trans-differentiation to brown or beige-like adipocytes.

\section{Directed differentiation of thermogenic adipocytes from pluripotent stem cells}

Only limited success has been reported for the directed differentiation of hPSCs to thermogenic adipocytes through a defined developmental pathway. In 2012, Saeki and colleagues serendipitously discovered that along with BMP7, hematopoietic factors promote the generation of thermogenic adipocytes from hPSCs [17]. Although BMP7 has been implicated in brown adipocyte development [13], hematopoietic factors have not and with the absence of detailed information about developmental origins, it is unclear if the cells reported developed through the classical brown adipocyte pathway. While the efficiency of thermogenic adipocyte specification was not clearly elucidated, these cells appeared to improve glucose clearance in rodent transplantation models. The field awaits confirmation of these findings but if this proves to be a reliable methodology it will significantly help in the understand of thermogenic adipocyte biology and will pave the way for therapeutic development. Using a different approach, Dani and colleagues used an embryoid body, serum-based approach to develop brown-like adipocytes from hPSCs [18, 19]. While the cells generated in these studies were not shown to be functionally thermogenic, they developed through a PAX3 progenitor state, suggesting that they have a paraxial mesoderm or dermomyotome origin. One point of contention here is that unlike in the mouse, expression of the skeletal muscle-brown adipocyte progenitor marker, MYF5, was not detected at a developmental stage anticipated from studies in mouse [20]. Because it is difficult to distinguish between beige and brown adipocytes based on marker gene expression alone, in the absence of anatomical information and detailed analysis of lineage development it remains possible that cells described by Dani and colleagues may be more representative of beige adipocytes. Several limitations are likely to prevent the direct utilization of hPSC-derived thermogenic cells in a clinical context with current technology. For example, low purity of cell populations and absence of stringent data demonstrating sustained thermogenic capacity following transplantation is an area that requires further attention.

Other recent studies have attempted to derive thermogenic adipocytes by differentiating hPSCs through a mesenchymal stem/progenitor cell [21]. These cells would therefore most closely resemble beige adipocytes, not brown adipocytes, and may be used for therapeutic studies in the process of 'browning'. In this approach, differentiated hPSCs were differentiated to a lateral plate mesoderm progenitor and then subsequently utilized a serumbased thermogenic adipose specification medium. These hPSC-derived beige adipocytes had all hallmarks of thermogenic adipocytes, such as UCP1 expression, high mitochondrial content, multi-locular lipid droplets and an increased oxygen consumption rate. While additional functional analyses and transplantation assays will need to be performed to fully evaluate the utility of these cells, this is the first report describing the establishment of beige adipocytes from hPSCs and can provides a useful starting point to further refine hPSC-tobeige adipocyte differentiation protocols. As an alternative to using iPSC models of beige adipogenesis, adipose-derived stem/stromal cells (ADSCs) may also be utilized (see below). This may be advantageous for several reasons, including a shorter differentiation timespan 
and the ability to isolate patient specific precursors by routine procedures. One disadvantage of ADSCs, however, is that unlike iPSCs they are not capable of long-term self-renewal. This makes some applications problematic, including limitations on the ability to routinely expand these cells for clinical applications.

One of the major limitations using hPSCs to derive brown or beige adipocytes relates to the low level of efficiency and reproducibility. Use of serum is a complicating factor and it will be necessary to follow developmental pathways in order to firmly establish reliable protocols. Specifically, serum is extremely complex in composition and is highly variable between batches. This often leads to inconsistent outcomes in the differentiation of pluripotent stem cells and adult stem cells [22, 23]. Careful comparison of hPSC-derived thermogenic cells to authentic human brown/beige fat is important, along with a complete functional characterization of the cells. Very few, if any, of the studies described above examined the efficiency of generating $\mathrm{UCP}^{+}$adipocytes. More rigorous experimentation will be necessary for any therapeutic approach to be pursued. All of this will be critical to identify a suitable cell that can be used for drug screening and disease modelling.

\section{Directed differentiation of thermogenic adipocytes from human adult stem cells}

The three most prominently used cell sources that enable the direct differentiation to brown or beige adipocytes are the hMADS2, Paz6 and SGBS cell lines [24-28]. Other groups have also reported the development of immortalized brown pre-adipocyte cell lines for this purpose [29]. Most of these cell lines display typical signatures of thermogenic adipocytes such as UCP1 expression and functionally demonstrated uncoupled mitochondrial respiration. Factors typically used to promote 'beiging' of hASCs include insulin [30], dexamethasone [31], rosiglitazone (BRL49653) [32] and triiodothyronine (T3) [33] in a serum-based medium. These factors are proposed to function by activating the thermogenic gene program through nuclear receptors, such as PPAR $\gamma$, thyroid hormone receptor and PGC1a.

Of these cell lines, hMADS2 has been the most extensively utilized to examine the molecular mechanisms underpinning conversion of hASCs to thermogenic adipocytes. One area of considerable interest has been the function of miRNAs during thermogenesis. As described previously, miRNAs may be important for BAT endocrine activity [13, 15, 16], but also have an important role in thermogenic adipocyte specification. Using the hMADS2 cell line Amri and colleagues uncovered a role for Let-7i-5p and miR-125b in suppressing beige cell formation. This was shown by using miRNA mimetics to suppress UCP1 expression in vitro and in vivo [34, 35]. Other studies also indicate that miR-26 is important for specifying thermogenic adipocytes by impacting the expression of the metalloprotease, ADAM17 [36]. The hMADS2 cell line has also been useful to examine the transcriptional regulatory networks regulating adipocyte browning. For example, the hMADS2 line was used to define transcriptional regulatory networks associated with PPAR $\boldsymbol{\gamma}$-bound super-enhancers that regulate the beige gene regulatory network [37]. Subsequently, KLF11, which is induced by $\operatorname{PPAR} \gamma$, was found to co-regulate this gene network. This study provides a clear example of how these cell lines can be used to understand the molecular mechanism underlying beige fat specification. 
As an alternative to using cell lines, other groups have used primary ADSCs, which typically have a mesenchymal stem cell signature, or brown pre-adipocytes to model thermogenic adipocyte differentiation [38, 39]. The caveat to using these cells is that they are incapable of sustained growth in culture which restricts their utility. Upon differentiation of these cells to brown or beige adipocytes, they acquire a gene expression profile associated with thermogenic adipocytes and functionally demonstrate uncoupled mitochondrial respiration. The most important potential utility of these cells is their use in the treatment of metabolic disease by autologous transplantation. Overall, these studies highlight the utility of primary and transformed adult stem cells in understanding how thermogenic adipocytes are formed and maintained. Furthermore, these studies suggest that these adult stem/progenitor cells and their differentiation to thermogenic adipocytes may provide utility to uncover novel diseasecausing mechanisms.

\section{Formation of thermogenic adipocytes by ectopic expression}

An alternative approach to derive thermogenic adipocytes involves the ectopic overexpression of transcription factors, either through the direct programming of hPSCs or through the re-programming of fibroblasts. In pioneering work, Cowan and colleagues induced the formation of thermogenic adipocytes through mesenchymal progenitor cells (MPCs) [40]. Embryoid bodies established from hPSCs served as a source of MPCs and then transcription factors known for their role in brown fat development (PPARG2, CEBPB and PRDM16) were ectopically expressed to drive a brown adipocyte-like cell fate. Importantly, these cells show many of the hallmarks of thermogenic adipocytes including high mitochondrial content, UCP1 expression and uncoupled mitochondrial respiration. Because these cells are not derived from a MYF5 ${ }^{+}$progenitor cells they do not recapitulate classical brown adipocyte development but instead, they seem to follow a pathway for beige fat development and could have utility in drug screening applications. One limitation of this approach, however, is that unlike the derivation of thermogenic cells from a defined differentiation with factors and small molecules, a trans-gene over-expression approach presents complications for use in a clinical setting. By taking a trans-differentiation strategy, Mazda and colleagues generated brown-like adipocytes from human and mouse fibroblasts [41]. Using ectopic expression of C/EBPB and MYC this work successfully generated beige-like adipocytes with high expression of UCP1 and uncoupled mitochondrial respiration. Importantly, transplantation of these cells into mice suppressed diabetes and obesity from high-fat diets, reduced circulating glucose levels and improved insulin sensitivity. Using different cell sources, these reports demonstrate proof-of-concept for using trans-gene over-expression to generate brown/beige-like cells that have potential utility for drug screening applications.

\section{Potential utility of thermogenic adipocytes for drug discovery and cell therapy}

There are two potentially synergistic approaches to utilize brown or beige fat cells as a therapeutic. The first is through a pharmacological approach that would target endogenous adipose stem cells (pre-adipocytes) or endogenous adipocytes (brown or beige) to induce their differentiation and/or thermogenic activation. In this approach, a transiently-acting 
chemical or drug that partially activates thermogenic activity would be potentially beneficial while minimizing undesirable effects associated with a sustained response and excessive activity. The second approach would be to transplant in vitro-derived brown or beige adipocytes that would serve to improve metabolic homeostasis. In this scenario, metabolic activation through pharmacological treatment would complement the therapeutic transplantation approach described previously. For either of these approaches to be successful, improvements to current cell culture models for brown or beige fat is required. For example, large numbers (>100 million) of cells would need to be produced in a Good Manufacturing Practice (GMP)-grade laboratory at purities far greater than that reported to date. Extensive functional analysis also needs to be performed to test safety and efficacy. In addition to the use of thermogenic adipocytes in a therapeutic context, brown/beige cells also provide an opportunity to model metabolic diseases in vitro. In this regard, both an hASC and hPSC models may prove beneficial, as both can be used to examine large cohorts of patient-derived cells.

\section{Identification of drugs and drug targets for the activation of thermogenic adipocytes}

One of the most important applications for increasing thermogenic adipocyte activity is in the treatment of patients with diabetes and related metabolic disorder, many of which are prevalent in the obese population. The identification of new drugs or drug targets would be critical for this purpose. Only a handful of cell-based chemical screens have been described however, that address this issue. Previously, Kajimura and colleagues developed a mouse model to establish a screening platform to identify new drugs that could activate brown adipocyte activity using a UCP1-luciferase reporter [42]. In addition to in vivo analysis of select drug compounds, brown pre-adipocytes were isolated and immortalized for cell-based screening of larger compound libraries. By performing a 300-compound library screen, the authors identified WWL113, which activated UCP1 activity [43]. While the exact target of WWL113 is unknown, it is likely to act by promoting PPARa signaling pathway, which is known to directly activate UCP1 expression [13]. Recently, Wolfrum and colleagues, performed a high-throughput image-based screen by coupling a mouse kinome inhibitor and shRNA library to identify kinases and signaling pathways important for mouse brown adipocyte development [43]. Using an immortalized mouse brown pre-adipocyte cell line, cells were differentiated to brown fat either after knock-down of kinases or with kinase inhibitors. To evaluate the differentiation or brown fat activation defects, automated imaging was performed to examine the quantity of lipid droplets or UCP1 staining. In total, nearly 200 kinases were identified that had a positive or negative impact upon brown fat proliferation or differentiation. The authors further evaluated one of the most promising targets, AMPK, and found that five of seven isoforms of the AMPK subunits regulate UCP1 levels. Several other studies have also described the importance of AMPK in metabolic diseases, which supports the notion that AMPK is a promising target for therapeutic intervention [44]. In the largest screen described to date, Ding and colleagues attempted to identify drugs that may reprogram the mouse myoblast cell line, $\mathrm{C} 2 \mathrm{C} 12$, to brown-fat like cells [45]. By screening more that 5 independent libraries and approximately 20,000 compounds the authors identified bexarotene, a selective RXR agonist without subtype specificity, as an inducer of brown adipogenic reprogramming from $\mathrm{C} 2 \mathrm{C} 12$ cells. In addition, to demonstrating the effect of bexarotene through RXR, bexarotene was also able 
to induce browning of subcutaneous WAT in vivo. This study highlights the importance of retinoids in brown fat development and potential therapeutic use.

In the only screen performed in human cells, Cowan and colleagues used the direct programming approach of hPSCs to brown adipocytes through the overexpression of PPARG2, CEBPB and PRDM16 to screen for small molecular that could substitute for CEBPB and PRDM16 [46]. Amongst the $>800$ molecules tested, JAK inhibitors were identified as compounds capable of activating UCP1 levels and subsequently shown to promote browning of white adipocytes by increasing multi-locular lipid droplets, endogenous UCP1 levels and mitochondrial respiration. Interestingly, JAK inhibition appeared to be operating through the suppression of interferon signaling which promoted the signaling of sonic hedgehog to transcriptionally activate UCP1. While the mechanism for this is not clear, it appears to operate independently from the cAMP/p38 pathway activated through $\beta 3$-adrenergic stimulation. Together, these studies highlight the utility of cell-based screens to identify new drugs and drug targets that regulate thermogenic adipocytes and have the potential to be used in a therapeutic setting. As most of the screens described thus far have been in mouse cells, further examinations of these drugs are needed in human cell culture models prior to clinical assessment. Importantly, differences have also been reported between mouse and human BAT, including their miRNA expression patterns, responsiveness to $\beta 3$-adrenergic signaling and their responses to glucocorticoids, which further supports the need to establish human drug screening models [47]. Further establishment and improvement in the human culture models of thermogenic adipocytes will be of substantial importance for future high-throughput or high-content screens.

\section{Transplantation-based therapeutics of thermogenic adipocytes}

A second important utility for human thermogenic adipocyte relates to their potential use in transplantation therapies. This concept is suggested by evidence in rodent models showing that transplantation of brown or beige fat has a positive impact on obesity and/or diabetes. For example, transplantation of BAT from donor embryos to diabetic NCRNU nude mice (a model for type I diabetes) reverses features of clinical diabetes and normalizes glucose tolerance [48]. BAT transplants also regulate glucose levels and insulin sensitivity in high-fat diet mouse models and reverses obesity in ob/ob mice [49, 50]. Importantly, transplantation of human beige adipocytes into animal models results in a similar response [51, 52]. Together, these data provide compelling evidence that transplantations of thermogenic adipocytes could ameliorate the effects of both type 1 and type 2 diabetes, improve wholebody glucose homeostasis, energy expenditure levels and potentially reverse obesity. These studies therefore open the opportunity for autologous cell-based beige fat transplants as a method to treat diabetes, obesity and other obesity-associated disorders. In this scenario, ADSCs could be isolated from patients then, programmed toward a beige cell fate and transplanted back into patient donors to alleviate disease. In this case, cells could be seeded into an encapsulation device to avoid the need for long-term immunosuppression treatment following transplantation [53]. 


\section{Modeling thermogenic adipocyte-associated diseases in a dish}

One final utility for developing robust brown or beige adipocyte differentiation models is to further understand the role of thermogenic adipocytes in various pathologies. For example, in the case of diabetes and obesity, patient cells can be isolated for the generation of hASCs and/or hPSCs and compared to normal individuals upon thermogenic adipocyte induction.

These adipocytes can be used to unravel the molecular mechanisms driving these diseases or potentially identify new therapeutic targets and biomarkers.

Maladaptive metabolism of adipose cells, where fat cells undergoes an incorrect metabolic response from a specific cue, can often complicate disease states. For example, lipodystrophy is often a side-effect of anti-retroviral therapy (ART) treatment in HIVinfected individuals. These HIV patients on ART may suffer from an altered flux of fatty acids or other substrates, low adipokine levels and altered glucose homeostasis [54]. In addition, studies now indicate that a decrease in beige thermogenic fat composition of subcutaneous WAT underlies lipodystrophy in HIV-ART treated patients [55]. Furthermore, their altered adipose tissues show reduced expression of thermogenic markers and reduced thermogenic activity $[55,56]$. These altered gene expression patterns may be directly tied to the loss of the miRNA processing machinery, such as Dicer, and result in reduced thermogenic differentiation or function. Cell-based transplantations of thermogenic adipocytes may provide a new opportunity for treating lipodystrophic HIV patients, as these transplants may improve glucose homeostasis and normalize substrate flux. Furthermore, stem cell-based thermogenic models may allow for the identification of novel browning agents that may be used in the context of ART.

In contrast to HIV-ART patients, other diseases or conditions may manifest maladaptive metabolic responses with increased levels of browning and accompanying thermogenic activity. Examples of this include cases of prolonged adrenergic stress, such as in severe burns [57, 58], or during metabolic dysfunction accompanying the wasting disorder, cancer cachexia [59]. For example, mice models of burns and human burn patients have been found to have increased mitochondrial content, increased UCP1 levels and decreased adiposity [57, 58]. In such cases, stem cell thermogenic models may help to identify new drugs that can suppress browning.

Cancer cachexia is characterized by severe adipocyte and skeletal muscle atrophy, resulting is significant weight loss and frailty and is associated with approximately $20 \%$ of all cancerassociated deaths [60]. In mouse models of cancer cachexia, studies have shown that the activation of brown fat or 'browning' of white fat as determined by increased UCP1 expression, energy expenditure or respiratory exchange ratios may occur [61, 62]. In tissue samples from patients with cancer cachexia display increased UCP1 levels [61], along with altered metabolic gene expression signatures [63]. Mechanistically, work from Spiegelman and colleagues has shown that the secretion of parathyroid-hormone-related protein (PTHrP) activates the thermogenic gene expression profile and suppression of this PTHrP-based signaling can suppress the fat and muscle atrophy associated with cancer cachexia [64, 65]. It should also be noted, however, that a UCP1-independent pathway has emerged as being important for white adipocyte atrophy associated with cancer cachexia [66, 67]. This is thought to operate through AMP-activated protein kinase and CIDEA [66, 67]. Conceivably, 
human stem cell thermogenic models of brown fat and 'browning' of white fat may be useful to examine the mechanisms underlying cancer cachexia. In this scenario, stem cells from cancer cachexia patients could be used to model the disease to see if there are any underlying genetic mechanisms that predispose individuals to cancer cachexia. These models may also be useful to identify drugs to suppress browning and potentially prevent cancer cachexia.

In the future, investigations that couple patient-cell modeling with large-scale genomic, transcriptomic, metabolic and proteomic analyses will provide insight into the molecular mechanisms that underpin pathologies from thermogenic adipocyte-associated diseases. While the undertaking of using human stem cells models to compare thermogenesis in normal and diseased patients has yet to be performed, we anticipate that this will be an important research area in the future.

\section{Conclusions and Future Perspectives}

Two major approaches have been described that can be used to derive thermogenic adipocytes; that is, directed differentiation and ectopic transcription factor expression. These approaches have various utilities for drug screening, cell therapy, disease modelling and understanding development. Despite these approaches, several improvements are required to fully utilize these cells in a clinical setting. For example, developing serum-free culture systems and improving the overall efficiencies and scalability of differentiation are critical (see outstanding questions box). In most cases, neither the direct quantitation of human thermogenic adipocyte differentiation and functionality, nor an improved energy expenditure following transplantation has been described, and so a careful, quantitative assessment between approaches needs to be performed.

Although these shortcomings need to be addressed, current evidence indicates that these approaches will be a powerful tool to treat a broad range of diseases that relate to metabolic dysfunction, with the treatment of metabolic disease such as type 2 diabetes and obesity being the highest priorities. One of the largest obstacles with cell replacement therapies is bypassing the patient's immune response to cell transplantation. One potential avenue here is the use of autologous ASCs for the generation of thermogenic adipocytes that can then be used for transplantation. The transplanted cells could be potentially encapsulated in an engineered device that can be monitored and replaced as necessary. This method been undertaken for hPSCs-derived islet cells for the treatment of type I diabetes [68]. The downside with any autologous transplantation approach is the significant cost of making thermogenic cells on a per-patient basis. In this regard, other approaches, such as using human pluripotent stem cells-derived brown or beige adipocyte that have HLA mutations, rendering them incapable of eliciting an immune response, may be feasible [69]. In this regard, an off-the-shelf thermogenic cell-type could be established for transplantations for potentially all patients and still avoid an immune response. Another important consideration here is the site of transplantation. For these cells to function efficiently, neovascularization must take place so that batokines can be effectively secreted, along with the facilitation of glucose clearance and fatty acid uptake. 
In sum, the identification of thermogenic adipocytes in adult humans and their implied therapeutic effect in obese individuals has established new opportunities for disease treatment and understanding disease pathogenesis of metabolic disorders. By taking advantage of thermogenic adipocyte human stem cell culture strategies, new drugs and drug targets may be identified for treatment of numerous diseases.

\section{Acknowledgments}

This work was supported by the National Institutes of Health, Institute of General Medical Sciences (P01GM085354).

\section{Glossary}

\section{Autologous Transplantation}

Patient cells are removed, potentially manipulated and then transplanted back to the same patient, thereby avoiding any immune response

\section{$\beta 3$-adrenergic}

One of the five main types of receptors signaling pathways in the autonomous nervous system that responds to neurotransmitters

\section{Cytokines/Batokines}

small secreted proteins that signal to surrounding cells. Batokines specifically refer to cytokines that are secreted from brown adipose tissue

\section{Dermomyotome}

A mesoderm-derived epithelial layer of the dorsal region of the somite that gives rise to skeletal muscle and brown fat

\section{Dexamethasone}

An anti-inflammatory synthetic adrenal corticosteroid that has been shown to promote adipogenesis

\section{Embryoid Body}

Spherical aggregates of pluripotent stem cells that can generate all germ layer lineages, thereby recapitulating early embryonic development

\section{Exosomes}

Vesicles of 30-100 nm in diameter that are secreted by many eukaryotic cell types

\section{Glucocorticoids}

Steroid hormones of the corticosteroid class that bind the glucocorticoid receptor and regulate gene expression

\section{Lipodystrophy}

A class of clinical disorders where the body is unable to produce adipose tissue

\section{Mesenchymal}


A multipotent stromal cell type that can generate bone, cartilage, muscle and adipocyte lineages

\section{Paraxial mesoderm}

A mesoderm sub-type that forms during early embryonic development and gives rise to the somites

\section{Pluripotent}

A cell type that is capable of giving rise to the three embryonic germ layers including mesoderm, endoderm and ectoderm

\section{Rosiglitazone}

A drug of the thiazolidinedione class that improves sensitivity to insulin and has been found to enhance 'browning' of white adipocytes

\section{Triiodothyronine}

A thyroid hormone that impacts numerous physiological processes such as differentiation and metabolism

\section{References}

1. Saely CH, et al. Brown versus white adipose tissue: a mini-review. Gerontology. 2012; 58(1):15-23. [PubMed: 21135534]

2. Lee P, et al. Brown adipose tissue in adult humans: a metabolic renaissance. Endocr Rev. 2013; 34(3):413-38. [PubMed: 23550082]

3. Kiefer FW. The significance of beige and brown fat in humans. Endocr Connect. 2017; 6(5):R70 R79. [PubMed: 28465400]

4. Cypess AM, et al. Identification and importance of brown adipose tissue in adult humans. $\mathrm{N}$ Engl $\mathrm{J}$ Med. 2009; 360(15):1509-17. [PubMed: 19357406]

5. Saito M, et al. High incidence of metabolically active brown adipose tissue in healthy adult humans: effects of cold exposure and adiposity. Diabetes. 2009; 58(7):1526-31. [PubMed: 19401428]

6. Virtanen KA, et al. Functional brown adipose tissue in healthy adults. N Engl J Med. 2009; 360(15): 1518-25. [PubMed: 19357407]

7. van Marken Lichtenbelt WD, et al. Cold-activated brown adipose tissue in healthy men. N Engl J Med. 2009; 360(15):1500-8. [PubMed: 19357405]

8. Lee YH, et al. In vivo identification of bipotential adipocyte progenitors recruited by beta3adrenoceptor activation and high-fat feeding. Cell Metab. 2012; 15(4):480-91. [PubMed: 22482730]

9. Shabalina IG, et al. UCP1 in brite/beige adipose tissue mitochondria is functionally thermogenic. Cell Rep. 2013; 5(5):1196-203. [PubMed: 24290753]

10. Wu J, et al. Beige adipocytes are a distinct type of thermogenic fat cell in mouse and human. Cell. 2012; 150(2):366-76. [PubMed: 22796012]

11. Inagaki $\mathrm{T}$, et al. Transcriptional and epigenetic control of brown and beige adipose cell fate and function. Nat Rev Mol Cell Biol. 2016; 17(8):480-95. [PubMed: 27251423]

12. Seale P. Transcriptional Regulatory Circuits Controlling Brown Fat Development and Activation. Diabetes. 2015; 64(7):2369-75. [PubMed: 26050669]

13. Villarroya F, et al. The Lives and Times of Brown Adipokines. Trends Endocrinol Metab. 2017

14. Markan KR, et al. Circulating FGF21 is liver derived and enhances glucose uptake during refeeding and overfeeding. Diabetes. 2014; 63(12):4057-63. [PubMed: 25008183]

15. Chen Y, et al. Exosomal microRNA miR-92a concentration in serum reflects human brown fat activity. Nat Commun. 2016; 7:11420. [PubMed: 27117818] 
16. Thomou T, et al. Adipose-derived circulating miRNAs regulate gene expression in other tissues. Nature. 2017; 542(7642):450-455. [PubMed: 28199304]

17. Nishio M, et al. Production of functional classical brown adipocytes from human pluripotent stem cells using specific hemopoietin cocktail without gene transfer. Cell Metab. 2012; 16(3):394-406. [PubMed: 22958922]

18. Hafner AL, et al. Brown-like adipose progenitors derived from human induced pluripotent stem cells: Identification of critical pathways governing their adipogenic capacity. Sci Rep. 2016; 6:32490. [PubMed: 27577850]

19. Mohsen-Kanson T, et al. Differentiation of human induced pluripotent stem cells into brown and white adipocytes: role of Pax3. Stem Cells. 2014; 32(6):1459-67. [PubMed: 24302443]

20. Sanchez-Gurmaches J, Guertin DA. Adipocyte lineages: tracing back the origins of fat. Biochim Biophys Acta. 2014; 1842(3):340-51. [PubMed: 23747579]

21. Guenantin AC, et al. Functional Human Beige Adipocytes From Induced Pluripotent Stem Cells. Diabetes. 2017; 66(6):1470-1478. [PubMed: 28270520]

22. Keller G. Embryonic stem cell differentiation: emergence of a new era in biology and medicine. Genes Dev. 2005; 19(10):1129-55. [PubMed: 15905405]

23. Yokoyama M, et al. Influence of fetal calf serum on differentiation of mesenchymal stem cells to chondrocytes during expansion. J Biosci Bioeng. 2008; 106(1):46-50. [PubMed: 18691530]

24. Elabd C, et al. Human multipotent adipose-derived stem cells differentiate into functional brown adipocytes. Stem Cells. 2009; 27(11):2753-60. [PubMed: 19697348]

25. Guennoun A, et al. Comprehensive molecular characterization of human adipocytes reveals a transient brown phenotype. J Transl Med. 2015; 13:135. [PubMed: 25925588]

26. Pisani DF, et al. Differentiation of Human Adipose-Derived Stem Cells into "Brite" (Brown-inWhite) Adipocytes. Front Endocrinol (Lausanne). 2011; 2:87. [PubMed: 22654831]

27. Zilberfarb V, et al. Human immortalized brown adipocytes express functional beta3-adrenoceptor coupled to lipolysis. J Cell Sci. 1997; 110(Pt 7):801-7. [PubMed: 9133667]

28. Wabitsch M, et al. Characterization of a human preadipocyte cell strain with high capacity for adipose differentiation. Int J Obes Relat Metab Disord. 2001; 25(1):8-15. [PubMed: 11244452]

29. Markussen LK, et al. Characterization of immortalized human brown and white pre-adipocyte cell models from a single donor. PLoS One. 2017; 12(9):e0185624. [PubMed: 28957413]

30. Tseng YH, et al. Differential roles of insulin receptor substrates in brown adipocyte differentiation. Mol Cell Biol. 2004; 24(5):1918-29. [PubMed: 14966273]

31. Zilberfarb V, et al. Effect of dexamethasone on adipocyte differentiation markers and tumour necrosis factor-alpha expression in human PAZ6 cells. Diabetologia. 2001; 44(3):377-86. [PubMed: 11317672]

32. Tai TA, et al. Activation of the nuclear receptor peroxisome proliferator-activated receptor gamma promotes brown adipocyte differentiation. J Biol Chem. 1996; 271(47):29909-14. [PubMed: 8939934]

33. Lee JY, et al. Triiodothyronine induces UCP-1 expression and mitochondrial biogenesis in human adipocytes. Am J Physiol Cell Physiol. 2012; 302(2):C463-72. [PubMed: 22075692]

34. Giroud M, et al. Let-7i-5p represses brite adipocyte function in mice and humans. Sci Rep. 2016; 6:28613. [PubMed: 27345691]

35. Giroud M, et al. miR-125b affects mitochondrial biogenesis and impairs brite adipocyte formation and function. Mol Metab. 2016; 5(8):615-25. [PubMed: 27656399]

36. Karbiener M, et al. MicroRNA-26 family is required for human adipogenesis and drives characteristics of brown adipocytes. Stem Cells. 2014; 32(6):1578-90. [PubMed: 24375761]

37. Loft A, et al. Browning of human adipocytes requires KLF11 and reprogramming of PPARgamma superenhancers. Genes Dev. 2015; 29(1):7-22. [PubMed: 25504365]

38. Bartesaghi S, et al. Thermogenic activity of UCP1 in human white fat-derived beige adipocytes. Mol Endocrinol. 2015; 29(1):130-9. [PubMed: 25389910]

39. Wang YL, et al. Concomitant beige adipocyte differentiation upon induction of mesenchymal stem cells into brown adipocytes. Biochem Biophys Res Commun. 2016; 478(2):689-95. [PubMed: 27498007] 
40. Ahfeldt T, et al. Programming human pluripotent stem cells into white and brown adipocytes. Nat Cell Biol. 2012; 14(2):209-19. [PubMed: 22246346]

41. Kishida T, et al. Reprogrammed Functional Brown Adipocytes Ameliorate Insulin Resistance and Dyslipidemia in Diet-Induced Obesity and Type 2 Diabetes. Stem Cell Reports. 2015; 5(4):56981. [PubMed: 26365511]

42. Galmozzi A, et al. ThermoMouse: an in vivo model to identify modulators of UCP1 expression in brown adipose tissue. Cell Rep. 2014; 9(5):1584-93. [PubMed: 25466254]

43. Perdikari A, et al. A high-throughput, image-based screen to identify kinases involved in brown adipocyte development. Sci Signal. 2017; 10(466)

44. Day EA, et al. AMPK as a Therapeutic Target for Treating Metabolic Diseases. Trends Endocrinol Metab. 2017; 28(8):545-560. [PubMed: 28647324]

45. Nie B, et al. Brown Adipogenic Reprogramming Induced by a Small Molecule. Cell Rep. 2017; 18(3):624-635. [PubMed: 28099842]

46. Moisan A, et al. White-to-brown metabolic conversion of human adipocytes by JAK inhibition. Nat Cell Biol. 2015; 17(1):57-67. [PubMed: 25487280]

47. Liu X, et al. Common and distinct regulation of human and mouse brown and beige adipose tissues: a promising therapeutic target for obesity. Protein Cell. 2017; 8(6):446-454. [PubMed: 28220393]

48. Gunawardana SC, Piston DW. Reversal of type 1 diabetes in mice by brown adipose tissue transplant. Diabetes. 2012; 61(3):674-82. [PubMed: 22315305]

49. Liu X, et al. Brown adipose tissue transplantation improves whole-body energy metabolism. Cell Res. 2013; 23(6):851-4. [PubMed: 23649313]

50. Stanford KI, et al. Brown adipose tissue regulates glucose homeostasis and insulin sensitivity. $\mathbf{J}$ Clin Invest. 2013; 123(1):215-23. [PubMed: 23221344]

51. Hepler C, et al. Directing visceral white adipocyte precursors to a thermogenic adipocyte fate improves insulin sensitivity in obese mice. Elife. $2017 ; 6$

52. Min SY, et al. Human 'brite/beige' adipocytes develop from capillary networks, and their implantation improves metabolic homeostasis in mice. Nat Med. 2016; 22(3):312-8. [PubMed: 26808348]

53. Orive G, et al. Cell encapsulation: technical and clinical advances. Trends Pharmacol Sci. 2015; 36(8):537-46. [PubMed: 26067102]

54. Grinspoon S, Carr A. Cardiovascular risk and body-fat abnormalities in HIV-infected adults. N Engl J Med. 2005; 352(1):48-62. [PubMed: 15635112]

55. Torriani M, et al. Dysfunctional Subcutaneous Fat With Reduced Dicer and Brown Adipose Tissue Gene Expression in HIV-Infected Patients. J Clin Endocrinol Metab. 2016; 101(3):1225-34. [PubMed: 26756119]

56. Mori MA, et al. Altered miRNA processing disrupts brown/white adipocyte determination and associates with lipodystrophy. J Clin Invest. 2014; 124(8):3339-51. [PubMed: 24983316]

57. Patsouris D, et al. Burn Induces Browning of the Subcutaneous White Adipose Tissue in Mice and Humans. Cell Rep. 2015; 13(8):1538-44. [PubMed: 26586436]

58. Sidossis LS, et al. Browning of Subcutaneous White Adipose Tissue in Humans after Severe Adrenergic Stress. Cell Metab. 2015; 22(2):219-27. [PubMed: 26244931]

59. Kir S, Spiegelman BM. Cachexia and Brown Fat: A Burning Issue in Cancer. Trends Cancer. 2016; 2(9):461-463. [PubMed: 28459108]

60. Fearon K, et al. Understanding the mechanisms and treatment options in cancer cachexia. Nat Rev Clin Oncol. 2013; 10(2):90-9. [PubMed: 23207794]

61. Petruzzelli M, et al. A switch from white to brown fat increases energy expenditure in cancerassociated cachexia. Cell Metab. 2014; 20(3):433-47. [PubMed: 25043816]

62. Tsoli M, et al. Activation of thermogenesis in brown adipose tissue and dysregulated lipid metabolism associated with cancer cachexia in mice. Cancer Res. 2012; 72(17):4372-82. [PubMed: 22719069]

63. Dahlman I, et al. Adipose tissue pathways involved in weight loss of cancer cachexia. Br J Cancer. 2010; 102(10):1541-8. [PubMed: 20407445] 
64. Kir S, et al. PTH/PTHrP Receptor Mediates Cachexia in Models of Kidney Failure and Cancer. Cell Metab. 2016; 23(2):315-23. [PubMed: 26669699]

65. Kir S, et al. Tumour-derived PTH-related protein triggers adipose tissue browning and cancer cachexia. Nature. 2014; 513(7516):100-4. [PubMed: 25043053]

66. Laurencikiene J, et al. Evidence for an important role of CIDEA in human cancer cachexia. Cancer Res. 2008; 68(22):9247-54. [PubMed: 19010897]

67. Rohm M, et al. An AMP-activated protein kinase-stabilizing peptide ameliorates adipose tissue wasting in cancer cachexia in mice. Nat Med. 2016; 22(10):1120-1130. [PubMed: 27571348]

68. Agulnick AD, et al. Insulin-Producing Endocrine Cells Differentiated In Vitro From Human Embryonic Stem Cells Function in Macroencapsulation Devices In Vivo. Stem Cells Transl Med. 2015; 4(10):1214-22. [PubMed: 26304037]

69. Gornalusse GG, et al. HLA-E-expressing pluripotent stem cells escape allogeneic responses and lysis by NK cells. Nat Biotechnol. 2017; 35(8):765-772. [PubMed: 28504668]

70. Hoene M, et al. The lipid profile of brown adipose tissue is sex-specific in mice. Biochim Biophys Acta. 2014; 1842(10):1563-70. [PubMed: 25128765]

71. Forner F, et al. Proteome differences between brown and white fat mitochondria reveal specialized metabolic functions. Cell Metab. 2009; 10(4):324-35. [PubMed: 19808025]

72. Sidossis L, Kajimura S. Brown and beige fat in humans: thermogenic adipocytes that control energy and glucose homeostasis. J Clin Invest. 2015; 125(2):478-86. [PubMed: 25642708]

73. An Y, et al. A Molecular Switch Regulating Cell Fate Choice between Muscle Progenitor Cells and Brown Adipocytes. Dev Cell. 2017; 41(4):382-391 e5. [PubMed: 28535373]

74. Bartelt A, et al. Brown adipose tissue activity controls triglyceride clearance. Nat Med. 2011; 17(2):200-5. [PubMed: 21258337]

75. Chondronikola M, et al. Brown adipose tissue improves whole-body glucose homeostasis and insulin sensitivity in humans. Diabetes. 2014; 63(12):4089-99. [PubMed: 25056438]

76. Orava J, et al. Different metabolic responses of human brown adipose tissue to activation by cold and insulin. Cell Metab. 2011; 14(2):272-9. [PubMed: 21803297]

77. Ouellet $\mathrm{V}$, et al. Brown adipose tissue oxidative metabolism contributes to energy expenditure during acute cold exposure in humans. J Clin Invest. 2012; 122(2):545-52. [PubMed: 22269323]

78. Hanssen MJ, et al. Short-term cold acclimation improves insulin sensitivity in patients with type 2 diabetes mellitus. Nat Med. 2015; 21(8):863-5. [PubMed: 26147760]

79. Laurila PP, et al. USF1 deficiency activates brown adipose tissue and improves cardiometabolic health. Sci Transl Med. 2016; 8(323):323ra13.

80. Kajimura S, et al. Brown and Beige Fat: Physiological Roles beyond Heat Generation. Cell Metab. 2015; 22(4):546-59. [PubMed: 26445512] 


\section{Highlights}

- Directed differentiation of human pluripotent and adult stem cells to thermogenic adipocytes are powerful models for human development.

- Thermogenic adipocytes generated by forward programming and reprogramming are potential platforms for drug discovery.

- Thermogenic adipocytes have utility for modeling of human metabolic disease and as a transplantable source of therapeutic cells. 


\section{Outstanding Questions}

- Will directed differentiation of hPSCs, guided by developmental principles, improve differentiation efficiencies so that brown adipocytes can be used therapeutically?

- $\quad$ Are there differences between brown and beige adipocytes, or the sources of these cells (pluripotent- or adult-derived), which may have an impact on drug discovery or cell therapy?

- Can exosomes from thermogenic adipocytes be used as a treatment for diabetes or obesity-associated diseases?

- $\quad$ Can any of these model systems result in new insights when comparing normal versus diseased individuals? 


\section{Box 1}

\section{Function and localization of thermogenic adipocytes}

Several differences exist between brown adipose tissue and white adipose tissue [1]. For example, white fat contains a large uni-locular lipid droplet while brown fat has multilocular lipid droplets of smaller size and variable fatty acids and lipid composition. Differences in lipid composition, which may also vary by sex, include increased levels of phospholipids lyso-phosphatidylcholine, phosphatidylcholine, lysophosphatidylethanolamine in brown fat and increased diacylglycerol and triacylglycerol in white fat [70]. Brown fat can also be distinguished from white fat by its increased mitochondrial volume. The mitochondrial proteome is widely different between white and brown fat, with brown fat having increased expression of proteins involved in the citric acid cycle, electron transport chain complexes I-IV and fatty acid metabolism, and decreased proteins involved in glycerolipid and fatty acid biosynthesis compared to white fat [71]. Most importantly, brown fat uniquely expresses UCP1 in the inner mitochondrial membrane. UCP1 allows for the leakage of protons back into the mitochondrial matrix through a proton conducting channel that is unlinked to the ATP synthase complex. This uncouples respiratory electron transport from ATP production. Since the energy that is generated is no longer used to fuel the ATP synthase turbine, in line with the $2^{\text {nd }}$ law of thermodynamics the energy is released as heat. This ability to generate heat serves a biologically critical function in hibernating and newborn animals, including humans. Functionally then, white fat is designed to store triglycerides for long-term energy generation while brown fat is engineered for thermogenesis [1].

Human BAT depots are found at several sites including the cervical, supraclavicular, paravertebral, para-aortic and peri-renal regions [3, 72]. Brown fat cells are derived from $\mathrm{MYF5}^{+}$progenitor cells that are descendants of embryonic paraxial mesoderm. MYF5 ${ }^{+}$ progenitors in the dermomyotome also generate skeletal muscle cells during embryonic development [73], indicating an important relationship between the two lineages. Beige fat, on the other hand is derived from $\mathrm{MYF5}^{-}$progenitors that give rise to ADSCs or preadipocytes [8-10]. Alternatively, beige cells can be derived from terminally differentiated white adipocytes following their acquisition of brown adipocyte characteristics. 


\section{Box 2}

\section{The Metabolic Sink Model}

Studied in rodents and humans indicate that brown adipose tissue (BAT) significantly improves the clearance of circulating glucose and triglycerides [74-77] and contribute to improved insulin sensitivity in type 2 diabetics [78]. Furthermore, patients with obesity associated co-morbidities such as heart disease may also find improvement from increased BAT activity [79]. The metabolic sink model proposes that increased thermogenic adipocyte activity functions as a sink for excessive levels of circulating metabolites, such as glucose and triglycerides [72, 80]. An outcome of increased brown/ beige fat activity in patients would be to potentially modulate levels of metabolites, improve whole-body metabolic homeostasis and increase energy expenditure. Therefore, it is postulated that through this mechanism, brown or beige fat may serve as an effective cell therapeutic for combating a wide-range of diseases such as obesity, type 2 diabetes, metabolic syndrome and cardiovascular disease. 


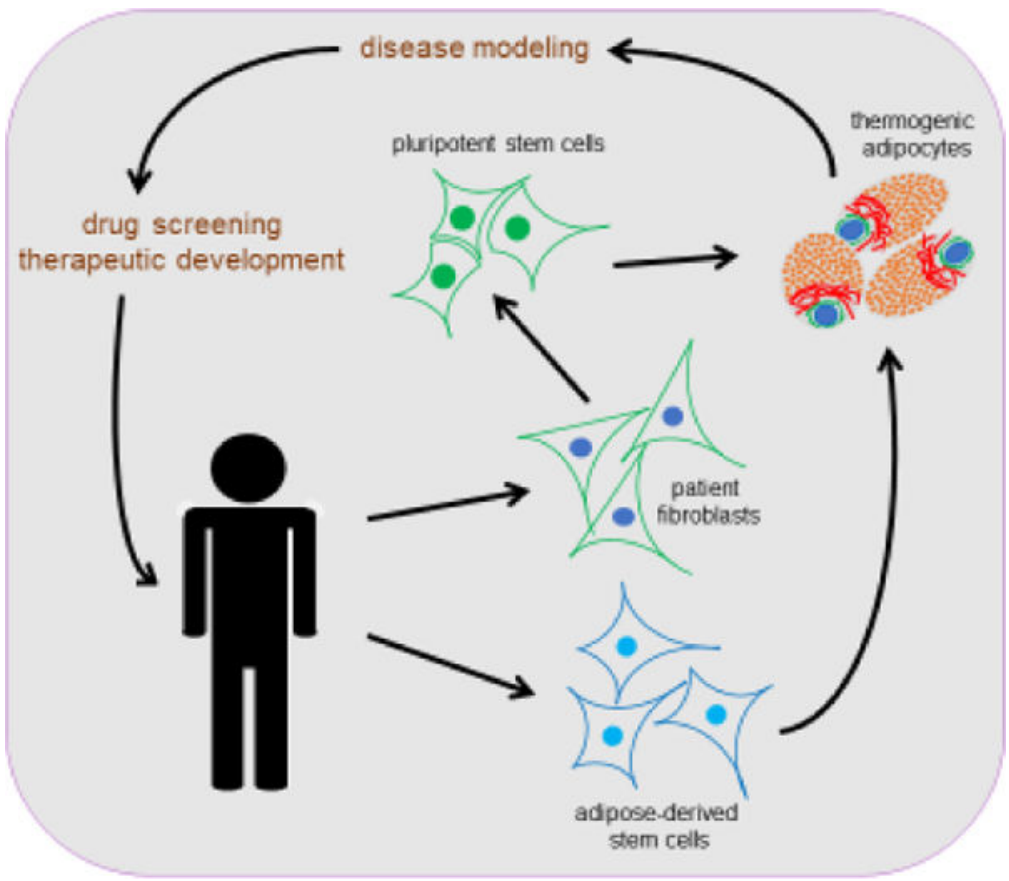

Figure 1. Human stem cells models of thermogenic adipocytes and their potential applications Cell-based models of thermogenic adipocytes can be generated from directed differentiation of stem cells, such as human pluripotent stem cells or adipose-derived stem/stromal cells. These thermogenic adipocytes are anticipated to be of important utility for disease modeling, cell therapies and drug screening applications. 


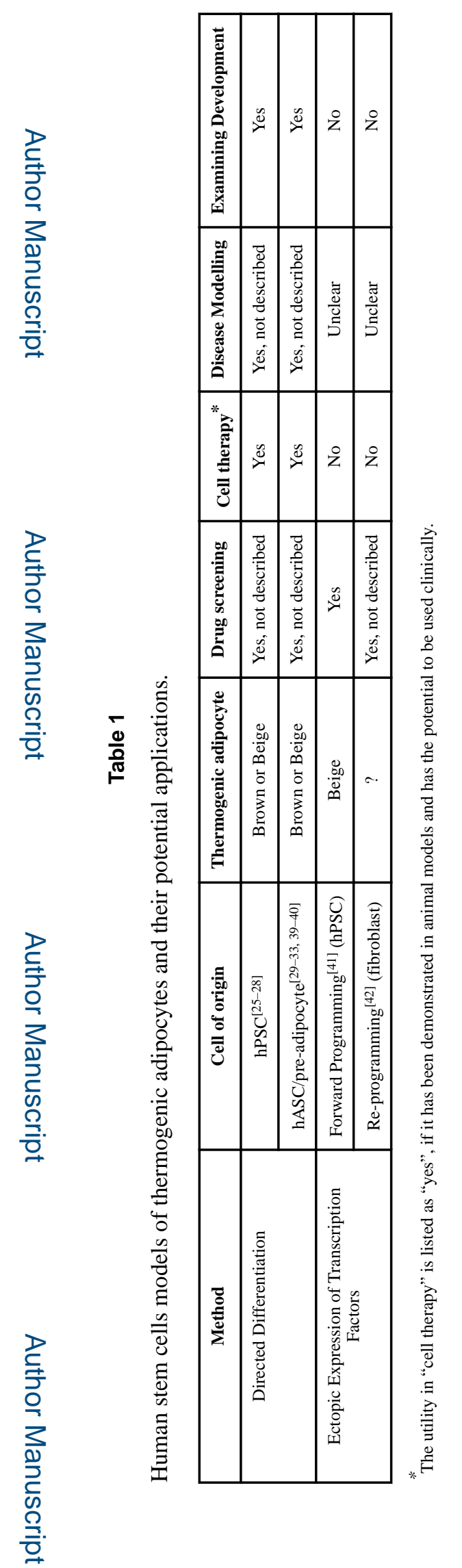

Trends Endocrinol Metab. Author manuscript; available in PMC 2019 May 01. 